\title{
The use of fetal exome sequencing in prenatal diagnosis: a points to consider document of the American College of Medical Genetics and Genomics (ACMG)
}

\author{
Kristin G. Monaghan, $\mathrm{PhD}^{1}$, Natalia T. Leach, $\mathrm{PhD}^{2}$, Dawn Pekarek, $\mathrm{MD}^{3}$, Priya Prasad, $\mathrm{MD}^{4}$ and \\ Nancy C. Rose, MD (i) ${ }^{5}$; on behalf of the ACMG Professional Practice and Guidelines Committee
}

\begin{abstract}
Disclaimer: This points to consider document is designed primarily as an educational resource for medical geneticists and other clinicians to help them provide quality medical services. Adherence to this points to consider document is completely voluntary and does not necessarily assure a successful medical outcome. This points to consider document should not be considered inclusive of all proper procedures and tests or exclusive of other procedures and tests that are reasonably directed to obtaining the same results. In determining the propriety of any specific procedure or test, the clinician should apply his or her own professional judgment to the specific clinical circumstances presented by the individual patient or specimen.

Clinicians are encouraged to document the reasons for the use of a particular procedure or test, whether or not it is in conformance with this points to consider document. Clinicians also are advised to take notice of the date this points to consider document was adopted, and to consider other medical and scientific information that becomes available after that date. It also would be prudent to consider whether intellectual property interests may restrict the performance of certain tests and other procedures.
\end{abstract}

Keywords: exome; prenatal diagnosis; fetal anomalies
Genetics in Medicine (2020) 22:675-680; https://doi.org/10.1038/s41436019-0731-7

\section{INTRODUCTION}

Approximately $2-4 \%$ of pregnancies are complicated by significant fetal structural anomalies. Given respect for reproductive autonomy, all patients diagnosed with a fetal anomaly should be offered genetic counseling, including review of options for genetic testing. ${ }^{1}$ The prenatal testing strategy and test selection should be individualized and guided by prenatal imaging findings and family history. Current options include chromosomal studies by karyotyping, fluorescence in situ hybridization, and chromosomal microarray analysis (CMA) with consideration of targeted gene-specific molecular testing for suspected disorders. A genetic diagnosis can assist in determining the fetal prognosis and inform prenatal care, including decisions on reproductive choice, in utero therapy, delivery planning, and neonatal management, potentially decreasing morbidity and mortality. It may also refine the recurrence risk leading to informed genetic counseling where future reproductive options, including preimplantation genetic testing, diagnostic prenatal testing, or consideration of donor gametes could be addressed.
Depending on the severity and number of anomalies, fetuses with sonographically identified structural birth defects will have up to a $30 \%$ likelihood of an abnormal karyotype., ${ }^{2,3}$ The higher resolution of CMA provides an additional diagnostic yield of $4-6 \%$ in fetuses with an ultrasound anomaly and normal karyotype. ${ }^{4,5}$ Thus, using these techniques, more than half of fetuses with structural anomalies remain without a diagnosis. Genome sequencing (GS) and exome sequencing (ES) are technologies that interrogate the genome at a nucleotide level. Genome sequencing involves assessing both the coding and noncoding regions of the genome, although a complete genome sequence is challenging to attain due to difficulty of sequencing and analysis in certain regions. ES is limited to the protein coding regions of more than 20,000 genes, comprising about $1-2 \%$ of the genome. Although GS may be more informative due to its scope, it requires greater data analytics and is not routinely utilized in clinical testing at this time. In contrast, ES either limited to the analysis of coding sequences of known disease-causing genes (clinical exome) or inclusive of genes of unknown clinical significance is a current option for the evaluation of fetuses with structural anomalies.

\footnotetext{
${ }^{1}$ GeneDx, Gaithersburg, MD, USA; ${ }^{2}$ Integrated Genetics, Laboratory Corporation of America, Westborough, MA, USA; ${ }^{3}$ Department of Obstetrics and Gynecology, Ochsner Health System, New Orleans, LA, USA; ${ }^{4}$ Department of Obstetrics and Gynecology, Wayne State University, Detroit, MI, USA; ${ }^{5}$ Department of Obstetrics and Gynecology, University of Utah, Salt Lake City, UT, USA.

Correspondence: ACMG (documents@acmg.net)

The Board of Directors of the American College of Medical Genetics and Genomics approved this statement on 18 November 2019.
} 
In pediatric and adult populations, clinical ES provides a diagnosis in $25-29 \%$ of individuals with disorders suggestive of a genetic etiology. ${ }^{6-8}$ Exome sequencing in the pediatric population is mostly used for children with multiple congenital anomalies, neurodevelopmental disorders, and seizures. Common indications for ES in the adult population include neurological, neuromuscular, oncologic, and cardiovascular disorders to determine the etiology of disease. ${ }^{8-11}$ Given the success in these patient populations, and the limitations of current genetic approaches to diagnoses, ES is now being applied in the clinical setting for fetuses with sonographic anomalies.

Initial reviews reported a prenatal ES diagnostic yield of up to $50-80 \% ;{ }^{12,13}$ however, these estimates were biased by small cohorts and highly selected cases. Two recent large-scale prospective studies reported the outcome for ES performed on chromosomally normal fetuses with structural anomalies. ${ }^{14,15}$ In the first study, Petrovski and colleagues performed trio ES on a cohort of 234 sequential pregnancies with unselected fetal structural anomalies detecting diagnostic variants in $10 \%$ of fetuses overall and in 19\% of fetuses with more than one anomaly. ${ }^{14}$ The detection rate was proportional to the severity of phenotype, with a range of $6 \%$ for fetuses with a single anomaly to $35 \%$ for fetuses with more than two anomalies. Another study by Lord et al. assessed a cohort of 610 fetus-parent trios. ${ }^{15}$ However, to ensure a wide range of anomalies, specific phenotypes were capped at $20 \%$ of the total number of fetuses. The ES trio approach with focused assessment of developmentally important genes achieved a genetic diagnosis in $8.5 \%$ of fetuses overall and in $15.4 \%$ of fetuses with more than one anomaly. Both studies demonstrate that ES increases the diagnostic yield in structurally abnormal fetuses by about 8-10\% after normal karyotype and CMA results, and the detection rate is strongly correlated with the number of fetal anomalies. The stringency of case selection, specifications of the testing platform, and availability of the parental data affect the diagnostic yield.

The analysis of ES data is phenotype driven, which is easier to ascertain in the adult or pediatric setting compared with the prenatal setting. Given that the assessment of the fetal phenotype is indirect, prenatal ES analysis could be limited to the reporting of variants in genes associated with the ultrasound findings. Accurate identification of fetal anomalies is dependent on the sophistication of fetal imaging equipment, maternal body habitus, fetal position, gestational age, and the experience of the health-care team. If fetal anomalies are highly suggestive of a specific diagnosis, single-gene testing or a phenotype-based gene panel may be more appropriate as the first-line test. However, for many fetal abnormalities, ES can be considered as an option when standard testing fails to identify a diagnosis.

The following points to consider document was developed with the intent to assist referring physicians, laboratory geneticists, genetic counselors, and other medical professionals in understanding the complexity and implications of ES testing as its utilization is increasing in prenatal care. This document is also intended to guide clinical laboratories in the development of appropriate protocols and policies as related to prenatal ES.

\section{POINTS TO CONSIDER}

\section{Pretest considerations}

- Exome sequencing may be considered for a fetus with ultrasound anomalies when standard CMA and karyotype analysis have failed to yield a definitive diagnosis. If a specific diagnosis is suspected, molecular testing for the suggested disorder (with single-gene test or gene panel) should be the initial test. At the present time, there are no data supporting the clinical use for ES for other reproductive indications, such as the identification of sonographic markers suggestive of aneuploidy or a history of recurrent unexplained pregnancy loss.

- Test design, including its genetic content, next-generation sequencing chemistry employed, and data analysis settings influence the overall test performance of ES. ${ }^{16}$ Laboratories should be transparent about methods and limitations of their testing platforms to aid clinicians' choice with regard to testing. Clinicians should seek guidance from the laboratory (or medical geneticist) regarding the methods and choice of available testing.

- Exome sequencing is a phenotype-driven test, therefore, the ordering health-care professional should provide the testing laboratory with adequate information required to generate the most accurate interpretation of results. Clinical information to be provided includes detailed fetal imaging reports such as sonograms, magnetic resonance imaging (MRI), and/or fetal cardiac ultrasound, prior fetal prenatal test results and/or clinical laboratory report, parental past medical history, ethnicity, reproductive history, and family history, including parental consanguinity.

- Trio analysis consisting of the proband and both biological parents is preferred to singleton (fetus only) or duo (fetus and one parent) analyses. Trio analysis consistently shows higher diagnostic yields compared with nontrio analysis. ${ }^{8}$ It allows for the immediate identification of de novo variants, determination of phase for biallelic variants, and confirmation of carrier status in both parents when a homozygous variant is detected. For laboratories not requiring trio analysis for prenatal ES, all efforts should be made to determine inheritance of identified fetal variants with targeted testing of the biological parents. ${ }^{17}$ There may be circumstances where both biological parents are unable to submit specimens. In this scenario, variant segregation testing using the available parent or testing of other closely related family members should be considered.

- Pretest counseling is ideally provided by a genetics professional during which the types of variants that may be returned in a laboratory report for all tested family members would be reviewed. Both pretest counseling 
and the informed consent process should also include the option to opt out of American College of Medical Genetics and Genomics (ACMG) defined secondary findings, incidental findings (i.e., pathogenic and likely pathogenic variants identified in genes unrelated to the test indication that are not part of the ACMG secondary findings gene list ${ }^{18,19}$ ), and the reporting of variants in nondisease genes. Counseling should also review that a negative report may be returned with this technology.

- With the use of prenatal ES, the turnaround time has to be rapid to maintain all aspects of reproductive choice. A rapid turnaround time has been demonstrated in the postnatal setting for critical genetic diagnoses in a pediatric and neonatal setting. ${ }^{20}$ Laboratories offering prenatal ES should have clearly defined turnaround times for this time-sensitive test.

- Sufficient specimen quantity is required for a rapid turnaround time, and ordering providers should be considerate of specimen requirements established by a testing laboratory.

- As with all prenatal genetic studies, the presence of maternal cell contamination that may interfere with the interpretation of fetal results must be excluded.

\section{Considerations for reporting}

While ES may increase the possibility of making a definitive diagnosis compared with single-gene and panel tests, this method also increases the likelihood of identifying variants of uncertain significance (VUS), incidental findings unrelated to the fetal phenotype and variants in the genes included in the ACMG secondary findings list. Molecular genetic testing of the fetus and biological parents can also identify misattributed parentage and undisclosed consanguinity. Laboratories should have clearly defined policies regarding the types of variants that will be reported for the fetus and parents and under what circumstances.

\section{Known disease genes}

- It is recommended that laboratories offering prenatal ES report pathogenic and likely pathogenic variants, as determined using ACMG variant interpretation guidelines in known disease genes consistent with the reported fetal phenotype. $^{18,21}$

- Laboratories may have different policies with regard to reporting VUS in a fetal specimen. Laboratories should consider reporting VUS in genes that fit the fetal phenotype, especially in autosomal recessive conditions if the VUS is found in trans with a pathogenic or likely pathogenic variant.

\section{Genes of uncertain significance}

- Variants in genes with a limited level of evidence for disease association (also referred to as candidate genes, genes of uncertain significance, or genes with limited evidence) may also be identified. These genes may have some evidence of being disease-associated based on a number of factors including internal laboratory data, animal models, or a limited number of published case reports. Any variant identified in a candidate gene should be classified no higher than VUS, regardless of the applicable ACMG criteria. ${ }^{21}$

- Laboratories should have clear policies regarding whether prenatal ES analysis and reporting is limited to only known disease genes or could include candidate genes and this information should be communicated to the individual(s) consenting to the test.

\section{Fetal incidental findings}

Incidental findings include variants in genes unrelated to the primary test indication that are not included on the ACMG secondary findings list. Incidental findings for the fetus could include clinically significant variants in genes associated with neurodevelopmental disorders, intellectual disability, or metabolic conditions that may not present with an ultrasound anomaly.

- Highly penetrant pathogenic variants detected in genes unrelated to the fetal phenotype, but known to cause moderate to severe childhood onset disorders, are recommended to be reported. Many of these disorders, especially those associated with nonsyndromic intellectual disability/neurodevelopmental disorders and metabolic conditions, are not detectable with fetal imaging.

- Regardless of ACMG variant classification, it is recommended that variants without a known fetal or childhood phenotype not be reported.

- Regardless of ACMG variant classification, it is recommended that fetal carrier status for autosomal recessive (male and female fetuses) and X-linked disorders (female fetuses) unrelated to the test indication not be reported.

\section{Parental incidental findings}

Incidental findings in the parental samples may include identification of carrier status for genetic conditions unrelated to the fetal phenotype or variants in genes known to cause late-onset conditions including neurological, neuromuscular, cardiovascular, or inherited cancer syndromes.

- Laboratories should have established policies for parental reporting of incidental findings as well as carrier status for autosomal recessive and $\mathrm{X}$-linked conditions. Of note, a laboratory's variant reporting, filtering, and analysis process could be set to limit the assessment of parental variants to only those present in the proband (fetus).

- If parental status for incidental findings are to be reported, we recommend it be limited to pathogenic and likely pathogenic variants. Reporting of incidental findings 
should be discussed with the patient as part of the pretest informed consent discussion and patients should be aware of their ability to opt out of the reporting of these variants.

\section{ACMG secondary findings}

- Secondary findings include the identification of clinically significant variants in genes for which ACMG recommends a return of results. Secondary findings should be discussed with the patient as part of the pretest informed consent discussion with the option of opting out of the reporting of these variants.

- If the patient consents to secondary findings analysis, variants identified in the ACMG secondary findings genes should be reported per current guidelines. ${ }^{19,22}$

- Laboratories should have clear policies regarding whether the reporting of secondary findings genes is limited to those variants identified in the fetus or will also include variants identified in either parent (irrespective of fetal inheritance). Of note, the laboratory's variant calling, filtering, and analysis process could be set to limit the assessment of parental variants to only those present in the proband (fetus).

\section{Misattributed parentage}

- Misattributed parentage can be identified with many genetic tests. This can be due to mistaken paternity or nondisclosure of a gamete donor. While this is not unique to ES, it is an important part of pretest counseling.

- Laboratories should have a defined policy regarding how misattributed parentage will be disclosed to the ordering health-care provider.

- For laboratories limiting fetal ES to trio-based analysis, misattributed parentage could result in the inability to complete testing if a specimen cannot be submitted by the biological parent expediently.

- For laboratories that do not require trio-based analysis, a policy should be established regarding how the exclusion from analysis of a submitted nonbiological parental specimen will be managed.

\section{Consanguinity}

- Bioinformatics can identify consanguinity between individuals undergoing ES. While parental consanguinity may complicate the analysis of ES due to an increased number of homozygous variants in the offspring, no degree of consanguinity renders ES impossible to complete.

- If the degree of relatedness is suggestive of a nondisclosed relationship between first or second degree relatives, standard recommendations should be followed. ${ }^{23}$

\section{Post-test considerations}

- Post-test counseling is recommended, regardless of the test result. It should be provided by individuals with relevant expertise, preferably a genetics professional.

- If a diagnosis is not achieved, prenatal care should be based on the available information from imaging. If a diagnosis is made, the findings may be used to guide further management. It should be emphasized that a negative prenatal exome result does not exclude a genetic diagnosis, and the results should not be used as reassurance of a normal outcome.

- Counseling should also include a discussion of the possibility of an upgrade or a downgrade of the classification of reported variants over time. In the event of a future pregnancy, variant reanalysis should be considered if prenatal testing is desired. ${ }^{24}$

\section{Consideration of cost}

- The cost effectiveness of prenatal ES is not known and is difficult to access accurately; estimates comparing ES with standard tests for pediatric neurodevelopmental delay project cost savings. ${ }^{12}$

- A potential financial benefit of prenatal diagnosis made by ES is eliminating the need for single-gene and gene panel tests when a specific diagnosis cannot be determined by prenatal phenotype that would direct specific gene testing. Cost savings may also be incurred with improved management plans for delivery; possible in utero treatment, eliminating possible metabolic risk factors to avoid a neonatal metabolic crisis; or recommendation for palliative care for a fatal prognosis.

- Additional tests that may be needed after prenatal ES include confirmatory imaging, biochemical and phenotypic assessment, and possibly parental evaluation for inherited autosomal dominant genes with variable expressivity.

- The cost of prenatal ES varies by laboratory as do billing policies and options for direct patient pay, payment plans, institutional billing, and insurance billing.

\section{Reanalysis considerations}

- For patients with initial negative ES results, reanalysis of exome sequencing data aids clinical diagnosis after 12 months. ${ }^{25}$ This outcome has been validated in the pediatric population as additional phenotypic findings might be noted during a child's growth and development. Continuous updates in database resources and new publications may provide further information for variant and gene classification.

- Due to the discovery of new gene-disease associations (that were unknown at the time of initial analysis), reanalysis can also be considered for diagnostic results and 
results deemed to be possibly (but not definitively) associated with the fetal phenotype.

- For fetal ES with nondiagnostic or negative results, reanalysis may be considered if a new phenotype develops in the proband after birth, a future pregnancy is planned, or a significant amount of time has passed (either at the discretion of the testing laboratory or at least 12 months) since the initial testing was performed. ${ }^{17,24}$

- If the original prenatal ES report does not account for the complete phenotype or if new/additional phenotypes develop over time, a reanalysis could be considered. ${ }^{26}$

- Laboratories should have a defined policy and protocol regarding the provision of ES reanalysis and the release of an updated report.

\section{Consideration for targeted family testing}

- The identification of pathogenic or likely pathogenic variants in a fetus enables carrier testing for other at-risk family members and possibly for future pregnancies.

- Laboratories should have policies regarding the availability of targeted testing for at-risk individuals. Couples receiving prenatal ES results that include variants classified as VUS should be made aware that future prenatal diagnosis with targeted testing and preimplantation genetic testing may be restricted by the laboratory's prenatal testing policy.

\section{Education of health-care professionals}

- As each patient's belief system is influenced by their social, religious, and cultural background in combination with their lifetime experiences, it is important to discuss expectations from genetic testing before making an informed decision. Various studies have highlighted the increased diagnostic value of genetic tests after careful patient selection by a trained genetics professional. ${ }^{27}$

- Health-care professionals should be educated regarding test characteristics including turnaround times, multifaceted costs, clinical utility, benefits, limitations, and future implications in terms of possible reanalysis before recommending ES as a test option. ${ }^{28}$

If unfamiliar with this technology, health-care professionals should seek appropriate resources or make a referral to a provider familiar with this testing.

- Studies have repeatedly emphasized the need for careful selection of patients for better diagnostic yield with ES. It is important to be aware of the associated costs and complex strategies needed to perform testing effectively. These include pre- and post-test genetic counseling, ultrasound scans from highly skilled sonographers experienced in fetal morphology, need for trio analysis in prenatal settings for better diagnostic capability, cost of manpower for variant interpretation and genetic data storage, multidisciplinary counseling dictated by the sequencing findings, and future new scientific findings requiring potential reanalysis and reinterpretation.

- If all laboratory modalities do not demonstrate an etiology for fetal anomalies, parents need to be counseled about the risk for recurrence, which could be low in case of a de novo pathogenic event, $25 \%$ if due to an autosomal recessive condition, or up to $50 \%$ for an autosomal dominant condition with reduced penetrance and/or variable expressivity.

- Given the use of trio analysis, it is possible that an incidental or secondary finding may be identified in a parent even if an etiology for the fetal presentation is not found. The option of including incidental and secondary findings must be clarified in pretest counseling. ${ }^{29}$

\section{CONCLUSION}

As a new diagnostic test in fetal medicine, ES may be considered when a diagnosis cannot be obtained using routine prenatal methods in a fetus with one or more significant anomalies. Data from prenatal ES may provide new information regarding the spectrum of anomalies in rare disorders or for well-established genetic conditions without known prenatal characteristics. It may also contribute to defining the clinical spectrum for lethal disorders, where the full phenotype may not yet be understood. Additional research is needed on patient perspectives of the consent process, effective and appropriate communication of uncertainty, return of results and reinterpretation, and health and economic outcomes. $^{29}$

\section{DISCLOSURE}

K.G.M. is an employee of GeneDx, Inc.; N.T.L. is an employee of Integrated Genetics, a specialty genetic testing group of Laboratory Corporation of America. The other authors declare no conflicts of interest.

Publisher's note Springer Nature remains neutral with regard to jurisdictional claims in published maps and institutional affiliations.

\section{REFERENCES}

1. Howe D. Ethics of prenatal ultrasound. Best Pract Res Clin Obstet Gynaecol. 2014;28:443-451.

2. Nicolaides KH, Snijders RJM, Gosden CM, Berry C, Campbell S. Ultrasonographically detectable markers of fetal chromosomal abnormalities. Lancet. 1992;340:704-707.

3. Zhang $S$, Lei $C, W u$ J, et al. A retrospective study of cytogenetic results from amniotic fluid in 5328 fetuses with abnormal obstetric sonographic findings. J Ultrasound Med. 2017;36:1809-1817.

4. Wapner RJ, Martin CL, Levy B, et al. Chromosomal microarray versus karyotyping for prenatal diagnosis. N Engl J Med. 2012;367:2175-2184

5. Hillman SC, McMullan DJ, Hall G, et al. Use of prenatal chromosomal microarray: prospective cohort study and systematic review and metaanalysis. Ultrasound Obstet Gynecol. 2013;41:610-620.

6. Yang Y, Muzny DM, Reid JG, et al. Clinical whole-exome sequencing for the diagnosis of Mendelian disorders. N Engl J Med. 2013;369: 1502-1511.

7. Lee $H$, Deignan JL, Dorrani $N$, et al. Clinical exome sequencing for genetic identification of rare Mendelian disorders. JAMA. 2014;312:1880-1887. 
8. Retterer K, Juusola J, Cho MT, et al. Clinical application of whole-exome sequencing across clinical indications. Genet Med. 2016;18:696-704.

9. Cukier HN, Dueker ND, Slifer SH, et al. Exome sequencing of extended families with autism reveals genes shared across neurodevelopmental and neuropsychiatric disorders. Mol Autism. 2014;5:1.

10. Bagnall $R$, Ingles J, Dinger $M E$, et al. Whole genome sequencing improves outcomes of genetic testing in patients with hypertrophic cardiomyopathy. J Am Coll Cardiol. 2018;72:419-429.

11. Shen T, Pajaro-Van de Stadt SH, Yeat NC, Lin JC. Clinical applications of next generation sequencing in cancer: from panels, to exomes, to genomes. Front Genet. 2015;6:1-9.

12. Best S, Wou K, Vora N, van der Veyver IB, Wapner R, Chitty LS. Promises, pitfalls and practicalities of prenatal whole exome sequencing. Prenat Diagn. 2018;38:10-19.

13. Vora NL, Powell $B$, Brandt $A$, et al. Prenatal exome sequencing in anomalous fetuses: new opportunities and challenges. Genet Med. 2017; 19:1207-1216.

14. Petrovski S, Aggarwal V, Giordano JL, et al. Whole-exome sequencing in the evaluation of fetal structural anomalies: a prospective cohort study. Lancet. 2019;393:758-767.

15. Lord J, McMullan DJ, Eberhardt RY, et al. Prenatal exome sequencing analysis in fetal structural anomalies detected by ultrasonography (PAGE): a cohort study. Lancet. 2019:393:747-757.

16. Rehm HL, Bale SJ, Bayrak-Toydemir P, et al. ACMG clinical laboratory standards for next-generiation sequencing. Genet Med. 2013;15: 733-747.

17. International Society for Prenatal Diagnosis, Society for Maternal and Fetal Medicine, and Perinatal Quality Foundation. Joint position statement from the International Society for Prenatal Diagnosis (ISPD), the Society for Maternal Fetal Medicine (SMFM), and the Perinatal Quality Foundation (PQF) on the use of genome-wide sequencing for fetal diagnosis. Prenat Diagn. 2018;38:6-9.

18. Strande NT, Riggs ER, Buchanan $A H$, et al. Evaluating the clinical validity of gene-disease associations: an evidence-based framework developed by the Clinical Genome Resource. Am J Hum Genet. 2017; 100:895-906
19. Green RC, Berg JS, Grody WW, et al. ACMG recommendations for reporting of incidental findings in clinical exome and genome sequencing. Genet Med. 2013;15:565-574.

20. Willig LK, Petrikin JE, Smith LD, et al. Whole-genome sequencing for identification of Mendelian disorders in critically ill infants: a retrospective analysis of diagnostic and clinical findings. Lancet Respir Med. 2015; 3:377-387.

21. Richards S, Aziz N, Bale $S$, et al. Standards and guidelines for the interpretation of sequence variants: a joint consensus recommendation of the American College of Medical Genetics and Genomics and the Association for Molecular Pathology. Genet Med. 2015;17:405-424.

22. Kalia SS, Adelman K, Bale SJ, et al. Recommendations for reporting of secondary findings in clinical exome and genome sequencing, 2016 update (ACMG SF v2.0): a policy statement of the American College of Medical Genetics and Genomics. Genet Med. 2017;19:249-255.

23. Rehder CW, David KL, Hirsch B, Toriello HV, Wilson CM, Kearney HM. American College of Medical Genetics and Genomics: standards and guidelines for documenting suspected consanguinity as an incidental finding of genomic testing. Genet Med. 2013;15:150-152.

24. Deignan JL, Chung WK, Kearney HM, Monaghan KG, Rehder CW, Chao EC. Points to consider in the reevaluation and reanalysis of genomic test results: a statement of the American College of Medical Genetics and Genomics (ACMG). Genet Med. 2019:21:1267-1270.

25. Ewans $L$, Schofield $D$, Shrestha $R$, et al. Whole-exome sequencing reanalysis at 12 months boosts diagnosis and is cost-effective when applied early in Mendelian disorders. Genet Med. 2018;20:1564-1574.

26. Clarke AJ. Managing the ethical challenges of next-generation sequencing in genomic medicine. Br Med Bull. 2014;111:17-30.

27. Committee on Genetics and the Society for Maternal-Fetal Medicine. Committee opinion no. 682 summary: microarrays and next-generation sequencing technology: the use of advanced genetic diagnostic tools in obstetrics and gynecology. Obstet Gynecol. 2016:128:e262-e268.

28. ACMG Board of Directors. Points to consider for informed consent for genome/exome sequencing. Genet Med. 2013;15:748-749.

29. Harris S, Gilmore K, Hardisty E, Lyerly AD, Vora NL. Ethical and counseling challenges in prenatal exome sequencing. Prenat Diagn. 2018;38:897-903. 